typeset using JPSJ.sty $<$ ver.1.0b $>$

\title{
Phenomenological theory of the 3 Kelvin phase in $\mathrm{Sr}_{2} \mathrm{RuO}_{4}$
}

\author{
Manfred SigRist and Hartmut Monien* \\ Yukawa Institute for Theoretical Physics, Kyoto University, Kyoto 606-8502, Japan
}

(Received October 27, 2018)

\begin{abstract}
We model the 3K-phase of $\mathrm{Sr}_{2} \mathrm{RuO}_{4}$ with Ru-metal inclusion as interface state with locally enhanced transition temperatures. The resulting $3 \mathrm{~K}$-phase must have a different pairing symmetry than the bulk phase of $\mathrm{Sr}_{2} \mathrm{RuO}_{4}$, because the symmetry at the interface is lower than in the bulk. It is invariant under time reversal and a second transition, in general, above the onset of bulk superconductivity is expected where time reversal symmetry is broken. The nucleation of the 3K-phase exhibits a "capillary effect" which can lead to frustration phenomena for the superconducting states on different Ru-inclusions. Furthermore, the phase structure of the pair wave function gives rise to zero-energy quasiparticle states which would be visible in quasiparticle tunneling spectra. Additional characteristic properties are associated with the upper critical field $H_{c 2}$. The $3 \mathrm{~K}$-phase has a weaker anisotropy of $H_{c 2}$ between the inplane and $z$-axis orientation than the bulk superconducting phase. This is connected with the more isotropic nature $\mathrm{Ru}$-metal which yields a stronger orbital depairing effect for the inplane magnetic field than in the strongly layered $\mathrm{Sr}_{2} \mathrm{RuO}_{4}$. An anomalous temperature dependence for the $z$-axis critical field is found due to the coupling of the magnetic field to the order parameter texture at the interface. Various other experiments are discussed and new measurements are suggested.
\end{abstract}

KEYWORDS: $\mathrm{Sr}_{2} \mathrm{RuO}_{4}, p$-wave superconductor, broken time-reversal symmetry, frustration effects, Andreev bound states

\section{§1. Introduction}

In recent years the quasi-two-dimensional metal $\mathrm{Sr}_{2} \mathrm{RuO}_{4}$ has advanced to one of the most intensely studied transition metal oxides displaying unconventional superconductivity. ${ }^{1,2)}$ There is strong evidence for spintriplet pairing with broken time reversal symmetry, a pairing state with the basic form $\mathbf{d}(\mathbf{k})=\hat{\mathbf{z}}\left(k_{x} \pm i k_{y}\right)$ (chiral p-wave state). ${ }^{3-5}$ ) This superconducting phase shows a variety of unusual properties among which the recently discovered "3-Kelvin" (3K) phase is one of the most puzzling findings. While the transition to the bulk superconducting state occurs around $1.5 \mathrm{~K}$, in samples with a large excess-Ru concentration a precursors to the superconducting transition appears at temperatures as high as $\left.3 \mathrm{~K} .{ }^{6,7}\right)$ This phase shows the features of an inhomogeneous superconducting phase. The detailed material analysis revealed that the excess-Ru does not distribute uniformly, but forms small inclusions of micrometer-size. Thus, the $3 \mathrm{~K}$-phase is likely connected with the phase separation of Ru-metal and $\mathrm{Sr}_{2} \mathrm{RuO}_{4}$ in this sample. The bulk superconductivity of metallic $\mathrm{Ru}$ has a superconducting transition temperature of $0.5 \mathrm{~K}$ only with a conventional ( $s$-wave) pairing state. This leads us to the assumption that the boundaries between the two materials provides the environment for the local nucleation of superconductivity at a higher temperature (Fig. 1).

It is not our aim to discuss here in detail the origin for the locally enhanced transition temperature, since the microscopic theory of superconductivity in $\mathrm{Sr}_{2} \mathrm{RuO}_{4}$ is

\footnotetext{
* Permanent address: Physikalisches Institut, Universität Bonn,
} Nussallee 12, 53115 Bonn, Germany still unclear. Nevertheless, we would like to comment on one important aspect which could be connected with the enhanced superconductivity. Various experiments have shown that a particular soft optical modes for local lattice distortion is associated with the inplane rotation of the $\mathrm{RuO}_{6}$-octahedra, the $\Sigma_{3}$-mode at the Brillouin zone boundary of the phonon spectrum, which leads to a slight volume reduction. ${ }^{8)}$ This rotation affects one of the three electron bands in particular, the $\gamma$-band which originates from the $4 d-t_{2 g}$-orbital with $d_{x y}$-symmetry. ${ }^{3,9)}$ While the dispersion for this orbital occurs via $\pi$-hybridization between the Ru- $d$-orbital and the O-p-orbitals in the undistorted case, the rotation introduces an additional $\sigma$ hybridization of opposite sign for the $d_{x y}$-orbital. A lattice distortion of this kind would diminish the dispersion of the $\gamma$-band and enhance the electron density of states, because of a Van Hove singularity near the Fermi level. ${ }^{10}$ ) It is likely that the $\mathrm{Ru}$-inclusions in $\mathrm{Sr}_{2} \mathrm{RuO}_{4}$ lead to internal stress that is released by local static distortions in the vicinity of the interfaces, most likely connected with the $\Sigma_{3}$-mode $\left(\hbar \omega_{\Sigma_{3}} \approx 140 \mathrm{~K}\right)$. A crude estimate from neutron scattering data yields a length scale of order 50 - $100 \AA$ over which this rotational distortion extend away from the interface. The increased density of states would lead to a locally enhanced $T_{c}$ independent of the microscopic mechanism. The additionally enhanced ferromagnetic spin fluctuations may support the spin-triplet pairing instability too. ${ }^{10)}$

In this paper we would like to investigate a number of properties of the inhomogeneous 3K-phase from a phenomenological point of view. A generalized GinzburgLandau (GL) formulation is most suitable for this purpose, since we will discuss an inhomogeneous supercon- 
ducting phase. The basic assumption is that the interface region has an enhanced transition temperature. We briefly review the basic conclusions of our theory. The first and most important fact is that the superconducting state nucleated at the interface has a different symmetry than the bulk superconducting phase. The interface superconducting state is time reversal symmetry conserving. Consequently, there is a further second order phase transition where this symmetry is broken. We will show that this transition occurs in general above the onset of bulk superconductivity. Naturally the nucleation of superconductivity at the interface is inhomogeneous and does not lead to a uniform phase transition. This is also true for the second transition. Even if all interfaces between $\mathrm{Ru}$-inclusions and $\mathrm{Sr}_{2} \mathrm{RuO}_{4}$ are locally equivalent, their geometry and mutual arrangement would lead to a spread on nucleation temperatures due to "capillary effects".

The $3 \mathrm{~K}$ superconducting state at the interface corresponds to an odd parity state, a $p$-wave state with a pair wave function that has a node parallel to the normal vector with a positive and negative lobes parallel to the interface (Fig. 1). This phase structure is responsible for a peculiar change of the quasiparticle spectrum due to Andreev reflection, i.e. the accumulation of Andreev bound states at zero-energy which may be observed by quasiparticle tunneling. The phase structure together with the topology of the interfaces can generate a frustration of the order parameter phase which can be released by introducing spontaneous orbital currents. Note that also the second transition to a time reversal symmetry breaking phase would reduce the frustration and introduce spontaneous currents.

Finally the upper critical field $H_{c 2}$ shows special properties in case of fields parallel to the interface. In this case the orbital depairing is reduced due to the essentially two dimensional nature of the condensate. In addition, the anisotropy of $H_{c 2}$ between fields parallel to basal plane of the strongly layered $\mathrm{Sr}_{2} \mathrm{RuO}_{4}$ and the $z$-axis direction is reduced compared with the critical field for the bulk phase. This is due to the more isotropic nature of the Ru-inclusions. A considerable fraction of the superconducting condensate of the $3 \mathrm{~K}$-phase resides in the Ru-part. An additional very striking feature occurs for magnetic field parallel to the $z$-axis of $\mathrm{Sr}_{2} \mathrm{RuO}_{4}$. While in zero field only the $p$-wave component with its node perpendicular to interface appears, the magnetic field drives also the other component whose node is parallel to the interface. The energy gain occurs via the coupling of the field to the current induced by the order parameter texture of both components. This coupling leads to a peculiar enhancement and temperature dependence of $H_{c 2}$ in agreement with experiment. ${ }^{6,7)}$

\section{§2. Phenomenological description}

The following analysis is based on the generalized GL theory as the most efficient way to describe basic properties of an inhomogeneous superconducting state. The bulk pairing state of $\mathrm{Sr}_{2} \mathrm{RuO}_{4}$ has the symmetry of a chiral $p$-wave state, represented by $\mathbf{d}(\mathbf{k})=\Delta_{0} \hat{\mathbf{z}}\left(k_{x} \pm\right.$ $\left.i k_{y}\right)$. This requires a two-component order parameter $\boldsymbol{\eta}=\left(\eta_{x}, \eta_{y}\right)$ with $\mathbf{d}(\mathbf{k})=\hat{\mathbf{z}}(\boldsymbol{\eta} \cdot \mathbf{k})$, which belongs to the two-dimensional representation $E_{u}$ of the tetragonal point group $D_{4 h}$ and describes the leading instability in $\mathrm{Sr}_{2} \mathrm{RuO}_{4}$. Note that recent flux distribution measurements in the mixed state have suggested the presence of two order parameter components. ${ }^{11)}$ On the other hand, $\mathrm{Ru}$ is a conventional s-wave superconductor with a transition temperature around $0.5 \mathrm{~K}$. The superconductivity in $\mathrm{Sr}_{2} \mathrm{RuO}_{4}$ penetrates the Ru-metal due to the proximity effect in two ways. First, proximity leads naturally to a spin-triplet pairing amplitude in $\mathrm{Ru}$, although its critical temperature there may be extremely small. Second, the spin-triplet superconducting state in $\mathrm{Sr}_{2} \mathrm{RuO}_{4}$ can induce the s-wave component. The corresponding coupling is, however, probably weaker than for the triplet channel because at the interface the triplet and singlet spin wave function have to be connected, by means of spin-orbit scattering. We will ignore the s-wave component in $\mathrm{Ru}$ and will briefly comment later only.

\subsection{Ginzburg-Landau free energy}

The GL free energy for the two-component order parameter $\boldsymbol{\eta}$ has the well-known form,

$$
\begin{aligned}
\mathcal{F}= & \int d^{3} r\left[a|\boldsymbol{\eta}|^{2}+b_{1}|\boldsymbol{\eta}|^{4}+\frac{b_{2}}{2}\left(\eta_{x}^{* 2} \eta_{y}^{2}+c . c .\right)\right. \\
& +b_{3}\left|\eta_{x}\right|^{2}\left|\eta_{y}\right|^{2}+K_{1}\left(\left|D_{x} \eta_{x}\right|^{2}+\left|D_{y} \eta_{y}\right|^{2}\right) \\
& +K_{2}\left(\left|D_{y} \eta_{x}\right|^{2}+\left|D_{x} \eta_{y}\right|^{2}\right)+\left\{K_{3}\left(D_{x} \eta_{x}\right)^{*}\left(D_{y} \eta_{y}\right)\right. \\
& +K_{4}\left(\left(D_{y} \eta_{x}\right)^{*}\left(D_{x} \eta_{y}\right)+c . c .\right\} \\
& \left.+K_{5}\left(\left|D_{z} \eta_{x}\right|^{2}+\left|D_{z} \eta_{y}\right|^{2}\right)+(\nabla \times \mathbf{A})^{2} / 8 \pi\right]
\end{aligned}
$$

where the coefficients are different in the two subsystems which we label by indices $\mathrm{S}$ and $\mathrm{R}$ for $\mathrm{Sr}_{2} \mathrm{RuO}_{4}$ and $\mathrm{Ru}$, respectively. The gradient terms contain the gauge invariant spatial derivatives $\mathbf{D}=\nabla+i(2 e / \hbar c) \mathbf{A}$ with $\mathbf{A}$ denoting the vector potential. The coefficients $K_{i \mu}$ determine the coherence length of the superconducting order parameter $\left(\mu=\mathrm{S}\right.$ and $\mathrm{R}$ ). Since $\mathrm{Sr}_{2} \mathrm{RuO}_{4}$ has a layered structure, the coherence length along the z-axis is short, $K_{5 S} \ll K_{1 S}, K_{2 S}, \ldots$ On the other hand, $\mathrm{Ru}$ is more isotropic so that $K_{5 R}$ has a magnitude similar to the other coefficients. In a weak-coupling approach assuming cylindrical or spherical Fermi surface shapes we obtain the relation $K_{1 \mu} / 3=K_{2 \mu}=K_{3 \mu}=K_{4 \mu}$. The second order coefficient $a_{\mu}(T)$ changes sign at the bare bulk transition temperature $T_{c \mu}$. For our discussion of the qualitative properties of the interface superconductivity it is sufficient to assume linear temperature dependence $a_{\mu}(T)=\alpha_{\mu}\left(T-T_{c \mu}\right)$ with $T_{c \mathrm{~S}} \approx 1.5 \mathrm{~K}$ and $T_{c \mathrm{R}}=0$.

For simplicity, we consider a single homogeneous planar interface with a normal vector parallel to the $x$-axis $(\mathbf{n}=(100))$ where $-\infty<x<0$ belongs to the Rumetal, and $0<x<\infty$ to $\mathrm{Sr}_{2} \mathrm{RuO}_{4}$. In this geometry the problem reduces to a one dimensional with the spatial direction along the $x$-axis. In the following we will always ignore the $z$-direction assuming homogeneity in this direction for simplicity. On the $\mathrm{Sr}_{2} \mathrm{RuO}_{4}$-side a thin layer of thickness $d$ with enhanced transition temperature is introduced. The boundary conditions, in general, involve reflection and transmission of Cooper pairs at 
the interface. We make the simplifying assumption of complete transparency, which corresponds to a continuous order parameter. We will, however, comment on the more general case below. We may describe the thin layer at the interface by a $\delta$-function in the free energy, if $d$ is much smaller than the coherence lengths along the $x$ axis (Fig. 1). Thus, the interface part of the free energy has the form,

$$
\mathcal{F}_{i}=\int d^{3} r \delta(x) \sigma|\boldsymbol{\eta}|^{2}
$$

where $\sigma=d \alpha_{\sigma}\left(T-T_{c \sigma}\right)$ with $T_{c \sigma}>T_{c \mathrm{~S}}$. This approximation is sufficient to discuss most important qualitative features of the $3 \mathrm{~K}$-phase.

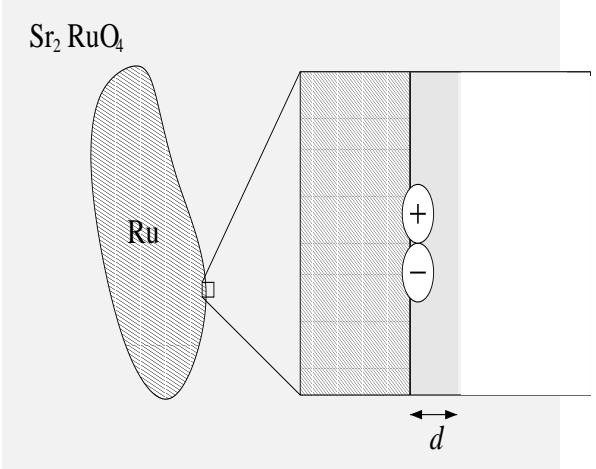

Fig. 1. Interface between $\mathrm{Sr}_{2} \mathrm{RuO}_{4}$ and a Ru-inclusion. The interface has a layer of thickness $d$ of enhanced transition temperature where a $p$-wave state nucleates whose wavefunction has the lobes parallel and the nodes perpendicular to the interface.

\subsection{Instability conditions of a planar interface}

We will first investigate the conditions of the nucleation of superconductivity at the planar interface. We consider a temperature range $T_{c \mathrm{~S}}<T<T_{c \sigma}$ where this local onset of superconductivity is supposed to occur. The instability condition is obtained by searching for the solution of the linearized GL equations which are given by

$$
\begin{gathered}
K_{1 \mu} \partial_{x}^{2} \eta_{x}-a_{\mu} \eta_{x}=0, \\
K_{2 \mu} \partial_{x}^{2} \eta_{y}-a_{\mu} \eta_{y}=0,
\end{gathered}
$$

for $x \neq 0(\mu=\mathrm{S}$ and $\mathrm{R}$ for $x>0$ and $x<0$, respectively). At $x=0$ the solutions have to be continuous and satisfy the following boundary conditions,

$$
\begin{gathered}
\left.K_{1 \mathrm{~S}} \partial_{x} \eta_{x}\right|_{x=0_{+}}-\left.K_{1 \mathrm{R}} \partial_{x} \eta_{x}\right|_{x=0_{-}}-\left.\sigma \eta_{x}\right|_{x=0}=0 \\
\left.K_{2 \mathrm{~S}} \partial_{x} \eta_{y}\right|_{x=0_{+}}-\left.K_{2 \mathrm{R}} \partial_{x} \eta_{y}\right|_{x=0_{-}}-\left.\sigma \eta_{y}\right|_{x=0}=0 .
\end{gathered}
$$

For temperatures above $T_{c \mathrm{~S}}$ the order parameter is largest at the interface and decays exponentially on both sides. For the $\eta_{x}$-component the solution is

$$
\begin{gathered}
\eta_{x}(x)=\eta_{x 0} e^{-x / \xi_{1 \mathrm{~S}}} \text { for } x>0 \\
\eta_{x}(x)=\eta_{x 0} e^{x / \xi_{1 \mathrm{R}}} \text { for } x<0
\end{gathered}
$$

with $\xi_{1 \mu}^{2}=K_{1 \mu} / a_{\mu}$. The analogous solution exists for $\eta_{y}$ with $\xi_{2 \mu}^{2}=K_{2 \mu} / a_{\mu}$. Then Eq.(5) and (6) lead to the instability equations,

$$
\begin{aligned}
& \sqrt{K_{1 \mathrm{~S}} a_{\mathrm{S}}}+\sqrt{K_{1 \mathrm{R}} a_{\mathrm{R}}}+\sigma=0 \\
& \sqrt{K_{2 \mathrm{~S}} a_{\mathrm{S}}}+\sqrt{K_{2 \mathrm{R}} a_{\mathrm{R}}}+\sigma=0,
\end{aligned}
$$

for $\eta_{x}$ and $\eta_{y}$, respectively. Because $K_{1 \mu}>K_{2 \mu}>$ $0\left(\xi_{1 \mu}^{2}>\xi_{2 \mu}^{2}\right)$ the nucleation will occur for the $y$ component. To obtain an instability temperature $T^{*}$ above $T_{c \mathrm{~S}}$ requires that

$$
\xi_{2 \mathrm{R}}\left(T_{c \mathrm{~S}}\right) a_{\mathrm{R}}\left(T_{c \mathrm{~S}}\right)<d \alpha_{\sigma}\left(T_{c \sigma}-T_{c \mathrm{~S}}\right) .
$$

This simple relation gives a good insight on the basic problem of the nucleation of local superconductivity at the interface. Obviously, the larger $d$ and the higher $T_{c \sigma}$, the higher is $T^{*}$. However, the presence of the normal-metal Ru tends to suppresses superconductivity, in particular if the coherence length $\xi_{2 \mathrm{R}}$ increases. Note that if both sides of the interface were $\mathrm{Sr}_{2} \mathrm{RuO}_{4}$, we always would find local superconductivity at a temperature higher than $T_{c \mathrm{~S}}$. Furthermore, the suppression by the Ru-metal is weaker, if the interface were less transparent leading to a discontinuity of the order parameter. Reduced transparency of the interface would not change the dominance of the $\eta_{y}$-component at this interface. The reason is that the order parameter component $\mathbf{n} \cdot \boldsymbol{\eta}$ is suppressed by a reflecting interface. In any case the degeneracy of $\eta_{x}$ and $\eta_{y}$ of the bulk region is lifted at the interface, since it corresponds to a region of effectively reduced symmetry. ${ }^{12)}$ The dominance of the $\eta_{y}$ component is also plausible from a microscopic point of view. The opening of a gap for momentum directions along the interface leads to the gain of condensation energy for quasiparticles with momenta parallel to the interface. These are the quasiparticles spending the longest time in the interface region.

We assume from now on that the condition Eq.(11) is satisfied and the resulting transition temperature is $T^{*} \approx 3 K$ corresponding to the $3 K$-phase. Note that the transition temperature does not depend on the orientation of the normal vector as long as it lies in the basal plane. The nucleating order parameter $\boldsymbol{\eta}$ is perpendicular to the normal vector, i.e., $\mathbf{n} \times \boldsymbol{\eta}$. The situation does not change much if a small $z$-axis components of the normal vector is introduced and the properties of the local superconducting state is determined by its inplane components.

Because the 3K-phase does not break time reversal symmetry in contrast to the bulk phase below $1.5 \mathrm{~K}$, a further second order phase transition has to occur at a temperature $T_{2}^{*}$ which is strictly larger than the bulk $T_{c}$. This is a different concept from conventional inhomogeneous superconductors, where superconductivity nucleates locally on small "islands" which with lowering temperature increase their overlap and, finally, form a bulk superconducting phase without further symmetry breaking (apart from the percolation transition). The temperature $T_{2}^{*}$ depends on the coupling between the order parameter components described by the fourth order terms in the free energy. The two pairing components 
may "attract" or "repel" each other, determined by the sign of the parameter $\tilde{b}=2 b_{1}-b_{2}+b_{3}(\tilde{b}>0$ repulsion and $\tilde{b}<0$ attraction). The weak coupling approach leads to $\tilde{b}>0$, which suppresses the appearance of the second component. Hence $T_{2}^{*}$ may be rather close to $T_{c}$ and difficult to distinguish experimentally from the bulk transition.

The discussion of the second transition and the form of the order parameter in the $3 \mathrm{~K}$-phase is complex as it involves also vector potential due to the presence of spontaneous currents in the time reversal symmetry-breaking phase. Thus we turn to the numerical solution of the complete set of GL equations including the complete set of GL equations for the order parameter and the vector potential. We use the parameters $\alpha_{\mathrm{S}}=\alpha_{\mathrm{R}}=\alpha_{\sigma}$ and $T_{c \sigma}=2.8 T_{c \mathrm{~S}}$. Except for $K_{5 \mu}$ we choose all coefficients $K_{j \mu}$ to be the same in $\mathrm{Ru}$ as well as in $\mathrm{Sr}_{2} \mathrm{RuO}_{4}$ : $K_{1 \mu} / \alpha_{\mu} T_{c \mathrm{~S}}=3 K_{i \mu} / \alpha_{\mu} T_{c \mathrm{~S}}=1(i=2,3,4)$, which corresponds to the basic zero-temperature coherence length $\xi_{0} \approx 1$. The coefficient for the $z$-axis gradient is small in $\mathrm{Sr}_{2} \mathrm{RuO}_{4}, K_{5 \mathrm{~S}} / \alpha_{\mu} T_{c \mathrm{~S}}=10^{-2}$, while we take, for simplicity, $K_{5 \mathrm{R}}=K_{2 \mathrm{R}}$, since $\mathrm{Ru}$ is more isotropic. The fourth order coefficient are also chosen independent of $x, 2 b_{1 \mu}=3 b_{2 \mu}=-3 b_{3 \mu}=0.4 \alpha_{\mu}$. We choose the interface layer thickness $d=1$, larger than it probably is in reality, for illustrative purpose and numerical stability. With these parameters we obtain a nucleation temperature of $T^{*} \approx 2 T_{c \mathrm{~S}}$ and $T_{2}^{*} \approx 1.33 T_{c \mathrm{~S}}$ which is rather high due to the large value of $d$. At this transition the $\eta_{x}$ component becomes finite in addition to $\eta_{y}$ and has the relative phase of $\pm \pi / 2$. In Fig. 2 we show the shape of the two order parameter components in the three different temperature regimes. Here a) represents the genuine $3 \mathrm{~K}$-phase with vanishing $\eta_{x}$-component (time reversal symmetry conserving phase), b) is the intermediate time reversal symmetry breaking state with both components finite. Finally, c) is the bulk superconducting phase of $\mathrm{Sr}_{2} \mathrm{RuO}_{4}$. Note, that both cases b) and c) possess a complex order parameter texture at the interface which will be important for the magnetic properties discussed in the next section.

\subsection{Capillary effect}

The interface instability for the superconducting state bears some resemblance with the wetting of a surface. ${ }^{13)}$ Similar to the wetting phenomena of liquids we find here capillary effects. Modulations of interfaces on length scales comparable to the coherence length can enhance the nucleation temperature. While this capillary effect is rather simple for conventional superconductors, there are complications in the case of an unconventional superconductor. In particular, there can be frustration effects due to the internal structure of the pair wave function, as we will see in the next section. Here we would like to consider first the rather simple situation of an interface that is not flat but has a weak modulation.

The enhancement of the transition temperature on a spatial modulated interface can be most simply interpreted by the effective increase of surface area. This corresponds also to an enhanced mutual overlap of the

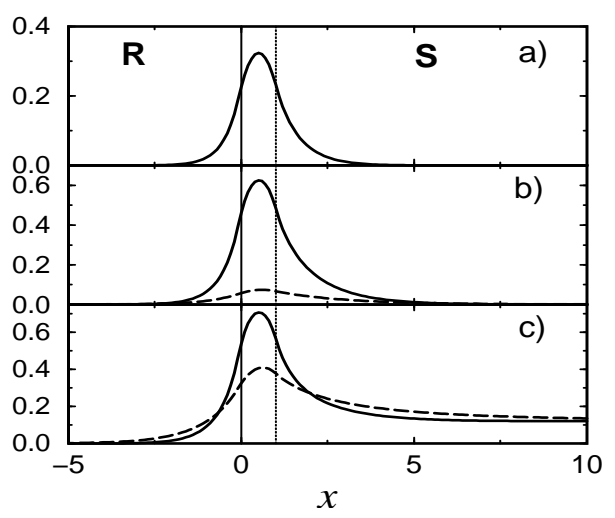

Fig. 2. Spatial dependence for the two order parameter components $\left(\left|\eta_{x}\right|\right.$ : dashed line and $\left|\eta_{y}\right|$ solid line): a) $3 \mathrm{~K}$-phase at $\left.T=1.75 T_{c \mathrm{~S}}>T_{2}^{*} ; \mathrm{b}\right)$ intermediate time reversal symmetry breaking phase at $T=1.25 T_{c \mathrm{~S}}<T_{2}^{*}$ with both components which have a relative phase $\pm \pi / 2$ ( $\rightarrow$ two-fold degeneracy); c) bulk superconducting phase $\mathbf{d}=\hat{\mathbf{z}}\left(k_{x} \pm i k_{y}\right)$ and a texture at the interface at $T=0.95 T_{c \mathrm{~S}}$.

order parameters nucleating at different points of the interface or on different inclusions. Let us assume that the interface is only slightly modulated, described by $x_{0}(y)=l \sin \left(2 \pi y / L^{\prime}\right)$ where $d \ll l \ll L^{\prime} \sim \xi_{i \mu}$. Then, by the most simple variational approximation we replace the interface term by

$\mathcal{F}_{i}=\int d^{3} r|\boldsymbol{\eta}|^{2} \sigma \sqrt{1+\left(\frac{2 \pi l}{L^{\prime}} \cos \left(\frac{2 \pi y}{L^{\prime}}\right)\right)^{2}} \delta\left(x-x_{0}(y)\right)$.

The important modification appears via the new interface metric, which accounts for the fact that the interface is wider or "denser". This leads to an enhanced instability temperature, even if we approximate the spatial dependence of the order parameter by a form like in Eq. $(7,8)$. Using this variation approach the instability equation for $\eta_{y}$ changes to

$$
\sqrt{K_{2 \mathrm{~s}} a_{\mathrm{S}}}+\sqrt{K_{2 \mathrm{R}} a_{\mathrm{R}}}+\sigma R=0,
$$

where the factor $R$ is

$R=\frac{1}{L^{\prime}} \int_{0}^{L^{\prime}} d y \sqrt{1+\left(\frac{2 \pi l}{L^{\prime}} \cos \left(\frac{2 \pi y}{L^{\prime}}\right)\right)^{2}} \approx 1+\left(\frac{\pi l}{L^{\prime}}\right)^{2}$.

Obviously, $R$ is always larger than 1 and leads to an effective enhancement of $\sigma$. We have ignored in our variational approach the spatial dependence of the order parameter along the interface. Including this aspect lead to even further enhancement of the nucleation temperature.

Another aspect of the capillary effect is the mutual influence of interfaces which we would like to consider on the example of two parallel interfaces. To be specific we assume a thin Ru-metal slab of thickness $L$ sandwiched between $\mathrm{Sr}_{2} \mathrm{RuO}_{4}$, again with normal vector parallel to the $x$-axis. Both interfaces have the same properties. Then it is easy to derive the instability equations taking the boundary conditions into account. There are two combinations of the nucleating order parameters on the two interfaces: a "bonding" and "antibonding" configuration which is even or odd, respectively, under reflection 
at the center of the slab. For the instability equations of the $\eta_{y}$-component we obtain,

$$
\sqrt{K_{2 \mathrm{~s}} a_{\mathrm{S}}}+\tanh \left(\frac{L}{2 \xi_{2 \mathrm{R}}}\right) \sqrt{K_{2 \mathrm{R}} a_{\mathrm{R}}}+\sigma=0
$$

for the bonding and

$$
\sqrt{K_{2 \mathrm{~s}} a_{\mathrm{S}}}+\operatorname{coth}\left(\frac{L}{2 \xi_{2 \mathrm{R}}}\right) \sqrt{K_{2 \mathrm{R}} a_{\mathrm{R}}}+\sigma=0
$$

for the antibonding combination. Obviously, we recover in both cases the original instability equation Eq.(10), if we separate the two interfaces far apart, $L \gg \xi_{2 \mathrm{R}}$. If, however, $L \sim \xi_{2 \mathrm{R}}$ then the second (positive) term is diminished (enhanced) and a higher (lower) transition temperature $T^{*}$ results in the case of bonding (antibonding). Naturally, the same kind of capillary effect occurs also in the inverse situation where a $\mathrm{Sr}_{2} \mathrm{RuO}_{4}$ slab is surrounded by Ru. The bonding combination of the two interface states just corresponds to the adjustment of the phases of two superconducting islands. The antibonding combination is equivalent to a phase difference of $\pi$ and is the energetically least favorable case. We will see in the next section that this aspect is important for frustration effects of the $3 \mathrm{~K}$ phase.

We can conclude that the onset of the $3 \mathrm{~K}$ phase is rather inhomogeneous on the interfaces as well, because the capillary effects have a strong influence on the nucleation of the superconducting order parameter.

\section{§3. Physical consequences}

\subsection{Spontaneous interface magnetism}

While the onset of superconductivity is visible in the reduction of electrical resistance, the significant features of second transition at $T_{2}^{*}$ are less easy to detect. Bulk superconducting double transitions are often observed through specific heat anomalies. Here the only significant anomaly, however, is seen at the onset of bulk superconductivity at $T_{c}=1.5 \mathrm{~K}$. Magnetic properties may, on the other hand, allow us to observe the onset of the superconducting phase at $T_{2}^{*}$ that violates time reversal symmetry. The solution of the full GL equations show that the below $T_{2}^{*}$ spontaneous supercurrents occur parallel to the interface. In Fig. 3 we show the magnetic field and current distribution at the interface for the case corresponding to Fig. 2b. There are two currents along the interface flowing in opposite direction as a result of the texture of the two order parameter components at the interface. Screening currents are weak due to absence of superconductivity in the bulk. Thus, the field resulting from the currents is strongly peaked on the interface and can even generate an overall finite magnetic flux. Since the flux is finite, it could be observed, in principle, by high-resolution magnetic microscopes, such as scanning Hall probes or a SQUID microscopes. Another sensitive probe for local magnetic field distributions is zero-field muon spin relaxation, which is expected to show an increase of the depolarization rate below $T_{2}^{*}$. However, if $T_{2}^{*}$ is very close to $T_{c}$ (as is very likely the case) then the signal will be obscured by the bulk superconducting state which has previously been observed by $\mu \mathrm{SR}$. ${ }^{5}$ The muon spin relaxation experiment would in any case give a dis- tinction between magnetic properties of the $3 \mathrm{~K}$-phase at $T^{*}$ and the bulk phase.

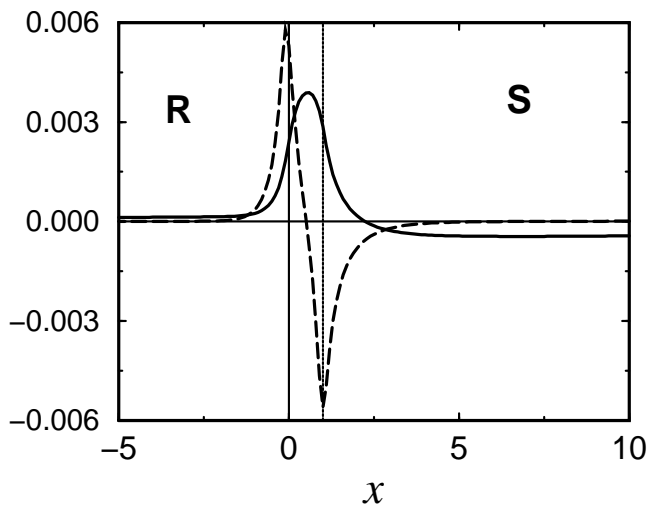

Fig. 3. Spontaneous magnetic field $B_{z}$ (solid line) and supercurrent $j_{y}$ (dashed line) distribution for the state given in Fig.2b) at $T=1.25 T_{c \mathrm{~S}}$.

\subsection{Frustration effects in the inhomogeneous state}

We have seen in the previous section on the capillary effect that the lowest energy configuration of order parameters nucleated on different interfaces naturally corresponds to equal order parameter phases. Let us now see how the superconducting state would arrange on several inclusions close enough to each other that their order parameters substantially overlap. If the order parameter were conventional, it would be easy to adjust all the order parameter phases on the different inclusion to be equal. This is not the case, if the order parameter has $p$-wave symmetry as is illustrated in Fig. 4 . The three Ru-inclusions depicted in Fig. 4 carry a superconducting state which on every point of the interface correspond to a $p$-wave state with the momentum direction aligned with the interface, i.e. the gap node lies always parallel to the normal vector. The interface regions on different inclusions close to each other behave like junctions or weak links connecting a network of the superconducting islands.

The direction of the positive lobe of the pair wave function is indicated by the arrows in Fig. 4 where we assume that the order parameter phase is constant on each inclusion. Parallel (antiparallel) arrows on neighboring interfaces correspond to bonding (antibonding) configurations of the overlapping order parameters, or equal phase ( $\pi$-shifted phases). It is obvious that in the arrangement of the order parameter on each inclusion introduces a $\pi$-phase shift between its weak links. Although we may change the phases on each island, it is impossible to adjust them so that all links have a zero phase difference for loops consisting of an odd number of inclusions (Fig. 4 shows this for the case of three inclusions). The situation is in many respects similar to the case described for granular $d$-wave superconductors. ${ }^{27,28,30)}$ We may identify loops which contain $\pi$ phase shifts and are consequently frustrated, since they cannot adjust the order parameter phase to minimize the energy of all weak links simultaneously. The frustration can be released by introducing 
phase gradients (supercurrents) which are energetically favorable, if the weak links grow strong enough. This would yield orbital magnetic moments. This effect results from the competition between weak link energy and magnetic field energy (determined by geometry) as in usual frustrated $\pi$-loops. However, the frustration also is reduced with the time reversal symmetry breaking transition at $T=T_{2}^{*}$. Both ways have similar impact by diminishing frustration and lead to spontaneous currents. In the network of superconducting islands the transition to the time reversal symmetry breaking state is inhomogeneous.

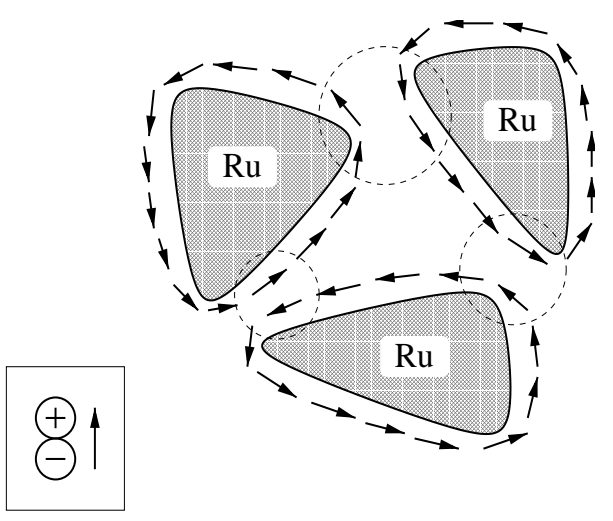

Fig. 4. Configuration of the $p$-wave pair wave function on $\mathrm{Ru}-$ inclusions (shadowed regions) in a crossection parallel to the $x$-y-plane. The arrow indicates the direction of positive wave function (see box). The dashed circles show the regions forming weak links between inclusions. The fact that the arrows of different inclusions in the weak link region are antiparallel corresponds to a phase difference of $\pi$. In the given configuration there is no way to adjust the phases of all three inclusions so that all weak links have minimal energy, i.e. a vanishing phase difference. Therefore this system is frustrated.

The frustrated 3K-phase with spontaneous currents can exhibit enhanced absorption in the ac-susceptibility. Dissipative processes, such as phase slips, are associated with the hysteretic reversal of the spontaneous currents. Thus the absorption would be sensitive to the application of a small static external fields which would bias the spontaneous currents (a similar situation was observed in granular high-temperature superconductors ${ }^{27,28,30)}$ ) The presence of spontaneous orbital magnetic moments in the inhomogeneous time reversal symmetry violating phase above $T_{c S}$ can cause a characteristic non-linear magnetic response in small external fields, similar to the paramagnetic Meissner effect or Wohlleben effect in high-temperature superconductors. ${ }^{27,28,30)}$ Under fieldcooling conditions the magnetic moments would be generated in a polarized way yielding a paramagnetic contribution. Probably, the signal would be considerably weaker than in high-temperature superconductors. The inhomogeneous 3K-phase is, however, more suitable to measure the spontaneous magnetism than the bulk superconducting phase, where the macroscopically visible magnetism is only resulting from surface currents, while it is screened or compensated in the interior of the systems.

\subsection{Quasiparticle spectrum}

The presence of superconductivity modifies the quasiparticle spectrum in small enclosed normal-metal regions through the formation of so-called Andreev bound states. Andreev bound states are standing waves of an electronhole superposition, e.g. in a normal metal region enclosed by a superconductor. In a quasiclassical picture the standing wave corresponds to an electron and hole travel on the same classical (ballistic) trajectory, but in opposite direction and are subject to Andreev reflection upon impact in the superconductor at both ends of the trajectory. The energy of such a standing wave depends on the phase of the gap functions at the boundaries of the classical trajectories and can be estimated by BohrSommerfeld quantization. Energies much smaller than the gap of the superconductor are approximately given by,

$$
E(n, \chi) \approx \frac{v_{F}}{2 L}[(2 n+1) \pi+\chi]
$$

where $v_{F}$ is the Fermi velocity, $L$ the length of the trajectory, $n$ an integer and, most important, $\chi$ is the difference between the phases of the gap functions at both ends of the ballistic trajectory (for simplicity we ignore here the effect of impurity scattering which can also be the origin low-energy states). For unconventional superconductors the phase difference $\chi$ depends also on the direction of the electron (hole) momentum, because the gap function, in general, has an anisotropic phase structure, i.e. a different phase for different directions of momenta on the Fermi surface. Thus, even in case of a homogeneous order parameter, the phase $\chi$ appearing in the Andreev scattering process would no trivial, but revealing the internal phase structure of the pairing state. For real order parameters we find $\chi= \pm \pi$ which is most important, because it leads to a zero-energy state independent of $v_{F}$ and $L$. This phase difference appears, if the gap function has positive and negative sign depending on the momentum direction on the Fermi surface. From the quasiclassical point of view underlying Eq.(17) the geometry of the classical trajectory decides about the phase $\chi=0$ or $\pi$. Since a large fraction of trajectories can have $\chi=\pi$ and all of them yield a zero-energy state, the zero-energy states $(\chi=\pi)$ lead to an enhanced density of quasiparticle states at the Fermi surface. The presence of this kind of zero-energy bound state has been intensively investigated for surfaces of $d$-wave supercondcutors. ${ }^{14,15)}$

In our case a $p$-wave pairing state nucleated at the interface generates a gap function which has regions of different sign on the Fermi surface. Zero-energy Andreev bound states are likely to occur within the Ru-inclusions as well as in $\mathrm{Sr}_{2} \mathrm{RuO}_{4}$ between $\mathrm{Ru}$-inclusions that are sufficiently close to each other. We encounter here a complex form of an (imperfect) Andreev billiard. The Andreev reflections are not perfect. Due the finite width of the superconducting regions, the zero-energy level acquires some width. Nevertheless, there is a strongly enhanced density of states at zero energy. Once time reversal symmetry is broken the phase differences $\chi$ for trajectories deviate from 0 and $\pi$. The originally enhanced density of states at zero-energy spreads over a larger en- 
ergy region. (Note that the reduction of the density of states at the Fermi energy represents also a driving force for the time reversal symmetry breaking transition, as was suggested also for the surface states of a $d$-wave superconductor. ${ }^{16,17)}$ )

One experimental indication for the enhanced density of states zero-energy states is the observation of so-called zero-bias anomaly, i.e. an increased quasiparticle tunneling conductance at zero voltage. Recent measurements of quasiparticle tunneling conductance in c-axis facing break junctions of $\mathrm{Sr}_{2} \mathrm{RuO}_{4}$ with $\mathrm{Ru}$-inclusions report the observation of zero-bias anomalies. ${ }^{18)}$ In the $3 \mathrm{~K}$-phase a zero-bias anomaly in current-voltage characteristics develops gradually with decreasing temperature. This conductance peak deforms into a pronounced bell shape combined with a residual zero-bias anomaly, below the bulk superconducting transition. Assuming that these tunneling features reflect the quasiparticle density of states connected with the Andreev billiard, we can interpret them within the scenario of the nucleated $p$-wave state which turns into a time reversal symmetry breaking state at lower temperature. We would like to emphasize the fact that break junctions in samples without $\mathrm{Ru}$ inclusions did not show any similar features above and below the onset of bulk superconductivity at $1.5 \mathrm{~K}$. Note also that an analogous phenomenon, the deformation of the zero-bias anomaly, has been observed for tunneling conductance into the $[110]$-surface of $\mathrm{YBa}_{2} \mathrm{Cu}_{3} \mathrm{O}_{7} \cdot{ }^{19}$ ) Also in that case broken time reversal symmetry is most likely cause. ${ }^{16,17)}$

It is clearly desirable to have more extensive experimental investigation of the quasiparticle spectrum in and around the Ru-inclusions via tunneling and related probes. $^{20-22)}$ In particular, using a contact to a single inclusion and measuring the quasiparticle currentvoltage characteristics through the Ru-metal inclusion into $\mathrm{Sr}_{2} \mathrm{RuO}_{4}$ could provide further valuable information on the $3 \mathrm{~K}$ as well as bulk superconducting phase. A detailed analysis of the phenomena discussed in this section will be given elsewhere.

\section{§4. Upper critical field}

The $3 \mathrm{~K}$ phase has an upper critical field which exceeds the bulk critical field considerably and shows a weaker anisotropy of in-plane versus out-of-plane critical field. The fact that superconductivity is confined in a small layer at the interface naturally leads to an reduction of orbital depairing, if the external magnetic field is applied parallel to the interface. This type of effect is known for superconductivity at other planar defects such as twin boundaries. ${ }^{23,24)}$ In a magnetic field superconductivity nucleates first at interfaces which are parallel to the field. We will analyze this effect for the [100]-interface with magnetic fields $\mathbf{B} \perp x$-axis. Unfortunately, a complete analytical treatment of this problem is not possible even for the GL formulation. Therefore we will restrict our analytic discussion to the region of very small fields at the onset of the $3 \mathrm{~K}$-phase and use a variational approach. This allows us to illustrate a few basic features of the upper critical field. Then we will consider the behavior of $H_{c 2}$ by numerical means for the model introduced above.
For temperatures close to $T^{*}$ the critical field is small and we may consider it as a perturbation. ${ }^{24)}$ Thus, we will use the exponential form of the order parameter appearing in zero-field at the onset of the 3K-phase: $\eta_{x \mu}=\eta_{x 0} \exp \left(-|x| / \xi_{1 \mu}\right)$ and $\eta_{y \mu}=\eta_{y 0} \exp \left(-|x| / \xi_{2 \mu}\right)$ with $\xi_{i \mu}=K_{i \mu} / a_{\mu}$. The magnetic field lies in the plane of the interface $\mathbf{B}=H(0, \sin \theta, \cos \theta)$ with the vector potential $\mathbf{A}=H\left(x-x_{0}\right)(0,-\cos \theta, \sin \theta)$. While in zerofield only the $\eta_{y}$-component is nucleated at $T^{*}$, in general both components can appear in a finite magnetic field. We use now the given form of the order parameter and calculate the free energy in a finite field by integrating over the spatial coordinates. In this first-order perturbative form the free energy per unit area of the interface is then up to second order in the the order parameter,

$$
\begin{aligned}
\mathcal{F}_{\text {var }}= & \sum_{\mu=\mathrm{S}, \mathrm{R}}\left[\left|\eta_{x 0}\right|^{2}\left\{a_{\mu} \xi_{1 \mu}+\frac{\sigma}{2}+\frac{\gamma^{2} H^{2}}{4} \xi_{1 \mu}^{3} f_{x \mu}(\theta)\right\}\right. \\
& +\left|\eta_{y 0}\right|^{2}\left\{a_{\mu} \xi_{2 \mu}++\frac{\sigma}{2}+\frac{\gamma^{2} H^{2}}{4} \xi_{2 \mu}^{3} f_{y \mu}(\theta)\right\} \\
& \left.+i\left(\eta_{x 0}^{*} \eta_{y 0}-\eta_{x 0} \eta_{y 0}^{*}\right) r \gamma H K_{2 \mu} \xi_{2 \mu}^{3} \cos \theta\right]
\end{aligned}
$$

with $\gamma=2 e / \hbar c$ and $r=(\sqrt{3}-1) /(\sqrt{3}+1)^{2}$ where we used the relation $\xi_{1 \mu}=\sqrt{3} \xi_{2 \mu}$, keeping the weakcoupling relations among different $K_{i \mu}$ for cylindrical symmetry. To simplify the free energy (18) we have fixed $x_{0}$ to zero, since it plays a minor role for our discussion. The anisotropy parameters are

$$
\begin{aligned}
& f_{x \mu}(\theta)=K_{2 \mu} \cos ^{2} \theta+K_{5 \mu} \sin ^{2} \theta, \\
& f_{y \mu}(\theta)=K_{1 \mu} \cos ^{2} \theta+K_{5 \mu} \sin ^{2} \theta .
\end{aligned}
$$

First we consider the case of the in-plane field $(\theta=$ $\pi / 2)$. Here only the $\eta_{y}$-component appears, which corresponds to the polar state with its nodes perpendicular to the field, which also appears in the bulk superconducting phase for in-plane fields. The zero of the corresponding coefficient in $\mathcal{F}_{v}$ defines the instability, which leads to

$$
H_{c 2}=\frac{\Phi_{0}}{\pi}\left[-d \frac{2 a_{y}^{*}(T)}{K_{5 R} \xi_{2 R}^{3}}\right]^{\frac{1}{2}}
$$

where we used that $K_{5 S} \ll K_{i \mu}, K_{5 R}(i \neq 5)$. In this case including a finite value of $x_{0}$ we find that $x_{0}=-\xi_{2 R} / 2$ maximizes $H_{c 2}$ (we omit here an explicit demonstration). Note that we can write $\sum_{\mu}\left(\xi_{2 \mu} a_{\mu}(T)+\sigma(T) / 2\right)=a_{y}^{*}(T)$ which is proportional to $\left(T-T^{*}\right)$ close to $T^{*}$. Consequently, we observe a square-root dependence $H_{c 2} \propto$ $\left|T^{*}-T\right|^{\frac{1}{2}} \cdot{ }^{23,24)}$ For curved interfaces the regions tangential to the field tend to allow the nucleation at higher temperature. Simultaneously, we have, however, to include capillary effects. Thus, the observation of a pure square-root temperature dependence would be masked in reality.

For the field parallel to the $z$-axis $(\theta=0)$ the $\eta_{x^{-}}$ component is involved too, despite the lower critical temperature in zero field. The optimal value of $x_{0}$ is very close to zero so that we fix $x_{0}=0$. With this simplification the instability equations involving the coupling of 


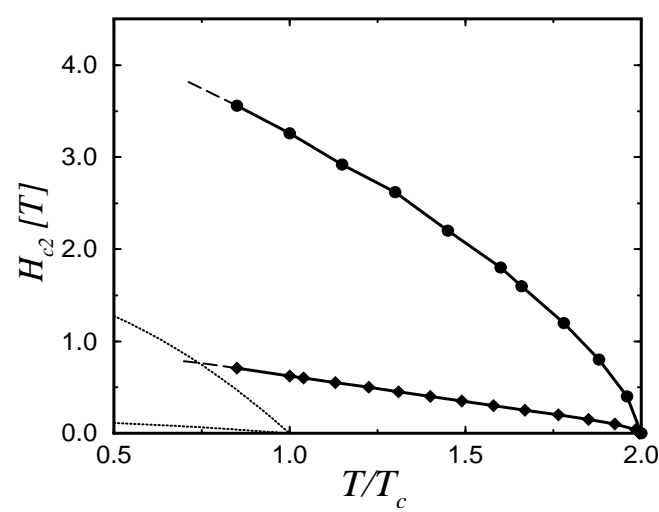

Fig. 5. Upper critical fields for the $3 \mathrm{~K}$ and bulk phase: The numerical solution of the GL equations give the upper critical fields of the 3K-phase: inplane (circles) and $z$-axis (diamonds). The corresponding bulk critical fields are given by doted lines. Note, the weaker anisotropy of the critical field of the $3 \mathrm{~K}$-phase. The numerical data compare well with the experimental data on a qualitative as well as quantitative level. 6,7 )

both components have the form,

$$
\begin{aligned}
& \left\{a_{x}^{*}(T)+\left(\frac{\gamma H}{2}\right)^{2} \Lambda_{x}\right\} \eta_{x 0}-i \gamma H \Lambda^{\prime} \eta_{y 0}=0 \\
& i \gamma H \Lambda^{\prime} \eta_{x 0}+\left\{a_{y}^{*}(T)+\left(\frac{\gamma H}{2}\right)^{2} \Lambda_{y}\right\} \eta_{y 0}=0
\end{aligned}
$$

where $\Lambda_{x}=\sum_{\mu} K_{2 \mu} \xi_{1 \mu}^{3}, \Lambda_{y}=\sum_{\mu} K_{1 \mu} \xi_{2 \mu}^{3}$ and $\Lambda^{\prime}=$ $r \sum_{\mu} K_{2 \mu} \xi_{2 \mu}$. Moreover, $a_{x}^{*}(T)=\sum_{\mu}\left(\xi_{1 \mu} a_{\mu}(T)+\right.$ $\sigma(T) / 2$ ) is the effective second order coefficient for the $\eta_{x}$-component and changes sign at a temperature $T_{c x}$ which lies between $T_{2}^{*}$ and $T^{*}$. The critical field is obtained by searching the non-trivial solution of this equation system,

$$
H_{c 2}(T) \approx \frac{\Phi_{0}}{\pi}\left[-\frac{a_{y}^{*}(T)}{\Lambda_{y}} \frac{a_{x}^{*}(T)}{a_{x}^{*}(T)-4 \Lambda^{\prime 2} / \Lambda_{y}}\right]^{\frac{1}{2}}
$$

where we used $\left|a_{y}^{*}(T)\right| \ll a_{x}^{*}(T)$ for $T \rightarrow T^{*}$. Thus we find again a basic square root behavior of $H_{c 2}(T)$ as in the case of inplane fields. However, in addition we see that the coupling of the two components yields an enhancement factor to $H_{c 2}$ which becomes stronger as the temperature is lowered. (Note, however, that the present form is only valid for $0<a_{x}^{*}(T) \gg 4 \Lambda^{\prime 2} / \Lambda_{y}$ and in any case our variational approach is applicable only in a restricted region close to $T^{*}$.) This enhancement is important, since it may modifies the overall form of the temperature dependence of $H_{c 2}$. Before considering this point numerically let us, however, address the issue of $H_{c 2}$ anisotropy.

For the bulk superconducting phase of $\mathrm{Sr}_{2} \mathrm{RuO}_{4}$ the critical fields are given by

$$
H_{c 2}^{\perp}=\frac{-\Phi_{0} a_{\mathrm{S}}(T)}{4 \pi K_{2 \mathrm{~S}}} \text { and } H_{c 2}^{\|}=\frac{-\Phi_{0} a_{\mathrm{S}}(T)}{2 \pi \sqrt{K_{2 \mathrm{~S}} K_{5 \mathrm{~S}}}}
$$

with $T<T_{c \mathrm{~S}}$ and ignoring anisotropy of $H_{c 2}$ for different inplane orientations, which is small. ${ }^{26)}$ This leads to an anisotropy factor of order $H_{c 2}^{\|} / H_{c 2}^{\perp} \approx 2 \sqrt{K_{2 \mathrm{~S}}} / \sqrt{K_{5 \mathrm{~S}}}$ whose experimental value is about 12 for $T$ close to
$T_{c \mathrm{~S}}$ in experiments $\left(K_{2 \mathrm{~S}} \gg K_{5 \mathrm{~S}} \approx 0.03 K_{2 \mathrm{~S}}\right){ }^{6)}$ On the other hand, the anisotropy for the $3 \mathrm{~K}$-phase may be less anisotropic with a factor $4 \sim 5,{ }^{6,7)}$ since

$$
\frac{H_{c 2}^{\|}}{H_{c 2}^{\perp}} \approx\left(\frac{2\left(\xi_{2 S}^{3} K_{1 S}+\xi_{2 R}^{3} K_{1 R}\right)}{\xi_{2 R}^{3} K_{5 R}}\right)^{\frac{1}{2}}
$$

gives a smaller ratio due to the fact that Ru-metal is basically isotropic with $K_{5 \mathrm{R}} \sim K_{2 \mu}$ and also the coherence lengths at $T=T^{*}$ are of the same order in $\mathrm{Ru}$ and $\mathrm{Sr}_{2} \mathrm{RuO}_{4}$. The reduction of anisotropy originates from the isotropy of the Ru-metal which leads to a stronger coupling of the order parameter to the inplane field than for $\mathrm{Sr}_{2} \mathrm{RuO}_{4}$.

The numerical evaluation of the upper critical field for the model used in Sect.2.2 illustrates the temperature dependence on qualitative level. The result are shown in Fig. 4 for the assumption that $K_{5 \mathrm{~S}}=0.03 K_{2 \mathrm{~S}}$ and $K_{5 \mathrm{R}}=K_{2 \mathrm{~S}}$. The circles and diamonds are the numerical results for the critical field inplane and along the $z$-axis, respectively. We observe indeed a weaker anisotropy for the critical field of the $3 \mathrm{~K}$-phase than for the bulk phase also indicate in Fig. 4. The initial temperature dependence has square root dependence as obtained analytically. While the inplane critical field has a downward curvature in the plotted temperature range, the $z$-axis field is nearly linear. The analytical expression in Eq.(22) indicates that even the upward curvature would be possible for the $z$-axis critical field. Our result is close to the experimental data qualitatively and to some extent quantitatively, with the choice of parameters giving $T^{*} \approx 2 T_{c}$ and the proper anisotropy of the bulk critical fields. ${ }^{6,7)}$ The peculiar temperature dependence of the $z$-axis critical field of the 3K-phase is also similar to that found in experiment. ${ }^{6,7)}$

\section{$\S 5$. The $s$-wave order parameter}

$\mathrm{Ru}$ metal becomes a conventional superconductor at a transition temperature of about $0.5 \mathrm{~K}$. Thus, we may include an s-wave order parameter into our theory. The coupling between the spin-singlet order parameter $\eta_{s}$ and the spin-triplet order parameter $\boldsymbol{\eta}$ requires that we take spin-orbit coupling into account. The difference between spin-orbit coupling in $\mathrm{Ru}$ and $\mathrm{Sr}_{2} \mathrm{RuO}_{4}$ yields spin flip tunneling at the interface which yields a coupling between $\eta_{s}$ and $\boldsymbol{\eta}{ }^{25,26)}$ The coupling term has the form

$$
\mathcal{F}_{\text {s.o. }}=\int_{\text {interface }} d S\left[\eta_{s}^{*}(\mathbf{n} \times \boldsymbol{\eta}+\text { c.c. }]\right.
$$

where $\eta_{s}$ is the $s$-wave order parameter on the Ru-side and $\boldsymbol{\eta}$ the two-component $p$-wave order parameter on $\mathrm{Sr}_{2} \mathrm{RuO}_{4}$-side, $\mathbf{n}$ is the interface normal vector and $t$ is a coupling constant. Obviously, for $\mathbf{n}=(100)$ only the $\eta_{y}$-component couples.

Further there is a coupling between the two order parameters also away from the interface which is again due to spin-orbit coupling. The corresponding term in the free energy, derived based on symmetry arguments, has the form,

$$
\mathcal{F}_{s p}=\int d V \tilde{a}\left[\left(D_{x} \eta_{y}-D_{y} \eta_{x}\right)^{*} \eta_{s}+c . c .\right]
$$


where $\tilde{a}$ is again a coupling constant different on the two sides. This term is only active, if there is a spatial variation of the order parameter or a magnetic field is present. We find that the spatial variation of the order parameter along the $x$-axis would couple exclusively the $\eta_{y}$-component to $\eta_{s}$.

This structure of order parameter coupling leads to a support for the nucleation of the $\eta_{y}$-component on the discussed [100]-interface due to the mixing with the $s$ wave order parameter intrinsic to $\mathrm{Ru}$. On a qualitative level, however, the inclusion of an $s$-wave component would not modify the properties of the $3 \mathrm{~K}$-phase on an essential way.

\section{§6. Conclusion}

In this article we have interpreted the $3 \mathrm{~K}$-phase of $\mathrm{Sr}_{2} \mathrm{RuO}_{4}$ with Ru-metal inclusion as an inhomogeneous superconducting state located at the interface between the material phases. This leads to a superconducting phase is different qualitatively from the bulk phase appearing below $1.5 \mathrm{~K}$. The $3 \mathrm{~K}$-phase conserves time reversal symmetry. This implies an additional phase transition where time reversal symmetry is spontaneously broken. This second transition occurs above the onset of bulk superconductivity. Irregular shapes and distribution of the $\mathrm{Ru}$ inclusions and the capillary effects would prevent a very sharp transition for the onset of the $3 \mathrm{~K}$ phase as well as the second transition.

The origin of multiple superconducting transitions is analogous to that of the splitting of the superconducting phase transition of degenerate order parameters by applying symmetry-lowering uniaxial stress. ${ }^{12,29)}$ Indeed the interface represents a region of the system where the symmetry is effectively lower, lifting the degeneracy between the two order parameters $\eta_{x}$ and $\eta_{y}$. Therefore the experimental proof of the conservation of time reversal symmetry in the $3 \mathrm{~K}$-phase would be a clear confirmation of a having a two-component order parameter.

We have argued that the phase structure of the pair wave function can lead to frustration effects in the coupling of the superconducting order parameters on different Ru-inclusions or different regions on the same inclusion. This is close related with the fact that this type of order parameter can lead to low- or zero-energy Andreev bound states, since the configuration of inclusions forms a complex Andreev billiard system. There are various experimental consequences due to these properties, some of which have been partially already investigated experimentally. (1) The Ru-metal inclusions provide an interesting way to tunnel into $\mathrm{Sr}_{2} \mathrm{RuO}_{4}$. Contacts via $\mathrm{Ru}$ inclusions could reveal more about the structure of the quasiparticle spectrum. ${ }^{18)}$ (2) The superconducting interface states of different neighboring $\mathrm{Ru}$-inclusions overlap and form a complex network. The study of the critical current as a function of temperature may give another tool to investigate the unconventional nature of this state. In particular, the frustration effects mentioned above and the spontaneous currents which occur in the time reversal symmetry breaking phase can yield characteristic anomalous behavior. (3) The lowfield magnetic response may show strong non-linear be- havior and cooling history-dependence in the time reversal symmetry breaking state for temperatures above $T_{c \mathrm{~S}}$, a phenomena which could be rather similar to the paramagnetic Meissner (or Wohlleben) effect in granular hightemperature superconductors. ${ }^{27,28,30)}$ (4) The study of microwave absorption in a small static magnetic field may show a non-monotonic field dependence as in some granular high-temperature superconductors. ${ }^{27,28,30)}$

These is only a selection of possible unusual properties of the 3K-phase. It is obvious that this phase and its properties provide a very good tool to investigate the superconductivity in $\mathrm{Sr}_{2} \mathrm{RuO}_{4}$ from a new point of view.

We would like to thank Y. Maeno, Z.Q. Mao, T. Akima, M. Wada, H. Yaguchi, Y. Liu and A. Furusaki for many stimulating discussions concerning the $3 \mathrm{~K}$ phase. This work was supported by a Grant-in-Aid of the Japanese Ministry of Education, Science, Culture and Sports. H.M. is also grateful for the support by a long-term fellowship of Japan Society for the Promotion of Science.

1) Y. Maeno et al.: Nature 372 (1994) 532. Physica C 282-287 (1997) 206.

2) T.M. Rice: Nature 396 (1998) 627.

3) T. M. Rice and M. Sigrist: J. Phys. Condens. Matter. 7 (1995) L643; G. Baskaran, Physica B 223-224 (1996) 490.

4) K. Ishida et al.: Nature 396 (1998) 658.

5) G.M. Luke et al.: Nature 394 (1998) 558.

6) Y. Maeno et al., Phys. Rev. Lett. 81 (1998) 3765.

7) T. Ando, T. Akima, Y. Mori and Y. Maeno, J. Phys. Soc. Jpn. 68 (1999) 1651.

8) M. Braden, W. Reichardt, S. Nishizaki, Y. Mori and Y. Maeno, Phys. Rev. B57 (1998) 1236.

9) T. Oguchi, Phys. Rev. B51 (1995) 1385; D.J. Singh, Phys. Rev. B52 (1995) 1358.

10) R. Matzdorf, Z. Fang, J. Zhang, T. Kimura, Y. Tokura, K. Terakura and E.W. Plummer, Science 289 (2000) 746.

11) P.G. Kealey et al., Phys. Rev. Lett. 84 (2000) 6094.

12) M. Sigrist, N. Ogawa and K. Ueda, J. Phys. Soc. Jpn. 60 (1991) 2341.

13) E. Montevecchi and J.O. Indeken, cond-mat/0009328.

14) C.R. Hu, Phys. Rev. Lett. 72 (1994) 1526.

15) Y. Tanaka and S. Kashiwaya, Phys. Rev. Lett. 74 (1995) 3451.

16) M. Matsumoto and H. Shiba, J. Phys. Soc. Jpn. 64 (1995) 1703; ibid. 3384; ibid. 65 (1996) 2194.

17) M. Fogelström, D. Rainer and J.A. Sauls, Phys. Rev. Lett. 79 (1997) 281.

18) Z.Q. Mao, K.D. Nelson, R. Jin, Y. Liu and Y. Maeno, condmat/0101410.

19) M. Covington, M. Aprili, E. Paraoanu and L.H. Greene, Phys. Rev. Lett. 79 (1997) 277.

20) M. Yamashiro, Y. Tanaka and S. Kashiwaya, Phys. Rev. B 56 (1997) 7847; J. Phys. Soc. Jpn. 67 (1998) 3224.

21) C. Honerkamp and M. Sigrist, J. Low Temp. Phys. 111 (1998) 895.

22) M. Matsumoto and M. Sigrist, J. Phys. Soc. Jpn. 68 (1999) 994.

23) A.I. Buzdin and L.N. Bulaevskii, JETP Lett. 34 (1981) 112; A.F. Andreev, JETP Lett. 46 (1987) 584.

24) A.A. Abrikosov, Fundamentals of the Theory of Metals, North-Holland, Amsterdam (1988).

25) V.B. Geshkenbein and A.I. Larkin, JETP Lett. 43 (1986) 395

26) M. Sigrist and K. Ueda, Rev. Mod. Phys. 63 (1991) 239.

27) P. Svedlindh, K. Niskanen, P. Nordling, P. Nordblad, L. Lundgren, B. Lönnberg and T. Lundström, Physica 162-164 (1989) 1365.

28) W. Braunisch, N. Knauf, G. Bauer, A. Kock, A. Becker, B. Freitag, A. Grütz, V. Kataev, S. Neuhausen, B. Roden, D. 
Khomskii and D. Wohlleben, Phys. Rev. B48 (1993) 4030.

29) M. Sigrist, R. Joynt and T.M. Rice, Europhys. Lett. 3 (1987) 629.

30) M. Sigrist and T.M. Rice, J. Phys. Soc. Jap. 61 (1992) 4283; Rev. Mod. Phys. 67 (1995) 503. 